\title{
ON NON-EXISTENCE OF RECTILINEAR MOTION IN PLASTIC SOLIDS AND NON-NEWTONIAN FLUIDS*
}

\author{
BY \\ D. E. STONE \\ Naval Research Laboratory
}

1. Introduction. For the class of materials described by Rivlin's theory of nonNewtonian fluids [1], Ericksen [2] has shown in the case of steady rectilinear motion that the speed is overdetermined unless the two scalars which occur in Rivlin's theory satisfy certain definite relations. When the speed is overdetermined, Ericksen conjectures that the only types of steady rectilinear motion possible are either rigid or such that the surfaces of constant speed are planes or portions of right circular cylinders. We show here that this conjecture is correct for the class of Rivlin fluids characterized by the requirement that the stress be unaffected by uniform changes in the speed. This requirement is one which is frequently imposed on theories of plasticity and in this case produces a theory very similar to the theory of perfectly plastic solids.

In addition, we consider the case of steady rectilinear motion through closed cylinders, wherein the speed is not overdetermined. Restricting our attention to the case in which the stress and speed are well behaved, we show that certain conditions on the behavior of the scalars which occur in Rivlin's equations must be satisfied in order that such a motion exist in every closed, smooth cylinder.

In the case of steady non-rectilinear motion through a tube of elliptical cross-section, Green and Rivlin [3] have obtained approximate solutions for a particular class of materials which are included in Rivlin's theory. Here, there is a rectilinear motion parallel to the axis of the tube on which is superimposed a vortex-like motion in each of the four quadrants of the tube's cross-section.

2. Equations of motion. In Cartesian tensor notation, Rivlin's equations are

$$
\begin{gathered}
t_{i i}=-p \delta_{i j}+F_{1}(\mathrm{II}, \mathrm{III}) d_{i j}+F_{2}(\mathrm{II}, \mathrm{III}) d_{i k} d_{k j} \\
t_{i i, i}+\rho h_{i}=\rho\left(\partial v_{i} / \partial t+v_{i, j} v_{i}\right) \\
v_{i, j}=0
\end{gathered}
$$

where $t_{i j}$ is the stress tensor, $d_{i j}$ is the rate of deformation tensor, defined in terms of the components of velocity $v_{i}$ by $d_{i j}=\frac{1}{2}\left(v_{i, i}+v_{i, j}\right), 2 \mathrm{II}=-d_{i j} d_{i j}, \mathrm{III}=\operatorname{det} . d_{i j}$, $h_{\boldsymbol{d}}$ is the body force per unit mass, $p$ is an arbitrary hydrostatic pressure, $F_{1}$ and $F_{2}$ are scalar functions of the indicated arguments, and $\rho$ is the density of the material which is assumed to be constant.

The analytic equivalent of the requirement that the stress be unaffected by uniform changes in the speed is that the function $f_{i j}\left(d_{k l}\right)=F_{1}$ (II, III) $d_{i j}+F_{2}$ (II, III) $d_{i k} d_{k l}$ satisfy the relation $f_{i i}\left(a d_{k l}\right)=f_{i j}\left(d_{k l}\right)$ for all $a>0$. In this event, it can be shown that the scalars $F_{1}$ (II, III) and $F_{2}$ (II, III) are of the form $G_{1}\left(\mathrm{III} /-\mathrm{II}^{3 / 2}\right)\left[-2 \mathrm{II}^{-1 / 2}\right]$ and $G_{2}\left(\mathrm{III} /-\mathrm{II}^{3 / 2}\right)[-2 \mathrm{II}]^{-1}$ respectively. Requiring that Eq. (3) hold, we have for the case of rectilinear motion where each particle moves parallel to the $x^{3}$ axis of a rectangular coordinate system at constant speed, $v_{1}=v_{2}=0, v_{3}=2 f\left(x^{1}, x^{2}\right)$; so that

*Received June 27, 1956. 
the quantities II and III are given by II $=-\left[f_{, 1}^{2}+f_{, 2}^{2}\right]$ and III $=0$. Restricting ourselves to the case in which $-I I>0$, we then have from Eqs. (1) and (2) respectively

$$
\begin{aligned}
\left\|t_{i i}\right\|=-p\left\|\delta_{i i}\right\| & +\frac{G_{1}(0)}{(-2 \mathrm{II})^{1 / 2}}\left\|\begin{array}{ccc}
0 & 0 & f_{.1} \\
0 & 0 & f_{.2} \\
f_{, 1} & f_{.2} & 0
\end{array}\right\| \\
& +\frac{G_{2}(0)}{[-2 \mathrm{II}]}\left\|\begin{array}{ccc}
f_{.2}^{2} & f_{.1} f_{.2} & 0 \\
f_{.1} f_{.2} & f_{.2}^{2} & 0 \\
0 & 0 & f_{.1}^{2}+f_{.2}^{2}
\end{array}\right\|
\end{aligned}
$$

and

$$
t_{i, i}+\rho h_{i}=0 .
$$

Assuming that the body forces $h_{i}$ are conservative so that $h_{i}$ can be replaced by $\Phi_{, i}$ and carrying out the differentiation called for in Eq. (5), we obtain the following three equations which describe the motion

$$
\begin{aligned}
& (p-\rho \Phi)_{, 1}=G_{2}(0)\left\{\left(f_{, 1}^{2}[-2 \mathrm{II}]^{-1}\right)_{, 1}+\left(f_{.1} f_{, 2}[-2 \mathrm{II}]^{-1}\right)_{, 2}\right\} \\
& (p-\rho \Phi)_{, 2}=G_{2}(0)\left\{\left(f_{, 1} f_{, 2}[-2 \mathrm{II}]^{-1}\right)_{, 1}+\left(f_{.2}^{2}[-2 \mathrm{II}]^{-1}\right)_{, 2}\right\} \\
& (p-\rho \Phi)_{, 3}=G_{1}(0)\left\{\left(f_{, 1}[-2 \mathrm{II}]^{-1 / 2}\right)_{, 1}+\left(f_{, 2}[-2 \mathrm{II}]^{-1 / 2}\right)_{, 2}\right\}
\end{aligned}
$$

Since $f$ is a function of $x^{1}$ and $x^{2}$ only and $G_{1}(0)$ and $G_{2}(0)$ are constants, it follows from Eqs. (6) that $p-\rho \Phi$ is of the form

$$
A x^{3}+X\left(x^{1}, x^{2}\right)
$$

If we assume that the rate of dissipation of energy, $t_{j}^{i} d_{i}^{i}$, is non-negative in the region of motion, then it follows from Eq. (4) that $G_{1}(0)$ is positive. When this is the case, the curvature of the curves of constant speed is constant, since the bracketed expression in the right-hand side of Eq. (6c) is the expression for the curvature of the curves $f\left(x^{1}, x^{2}\right)=$ constant [4]. We note here that this result depends only on the form of $F_{1}(\mathrm{II})$; namely, $K[-\mathrm{II}]^{-1 / 2}$ where $K$ is a positive constant.

3. The case $G_{2}(0)=0$. When $A \neq 0$, we obtain a solution for any function $f\left(x^{1}, x^{2}\right)$ for which the curves $f\left(x^{1}, x^{2}\right)=$ constant are circles of constant radius. For example, the function $f\left(x^{1}, x^{2}\right)=\left[x^{2}+\left(r_{0}^{2}-x^{12}\right)^{1 / 2}-r_{0}\right]$ gives a solution in the region bounded by the planes $x^{1}=-r_{0}$ and $x^{1}=r_{0}$. Outside of this region the curves $f\left(x^{1}, x^{2}\right)$ clearly intersect so that the solution is meaningless. It is easily seen in such a motion that the material cannot adhere to the wall of a closed rigid cylinder.

When $A=0$, the curves $f\left(x^{1}, x^{2}\right)=$ constant are straight lines (not necessarily parallel), and any function which has this property will give a solution. The most common example of motion of this type is the case of simple shear wherein the curves of constant speed are parallel straight lines.

4. The case where $G_{2}(0) \neq 0$. We show here that the curves of constant speed are parallel or concurrent straight lines, where we have assumed, as in the case $G_{2}(0)=0$, that - II $>0$.

Let us make the coordinate transformation $f^{i}=f^{i}\left(x^{1}, x^{2}\right)$, where the curves $f^{1}=$ 
constant are the curves of constant speed and the curves $f^{2}=$ constant are their orthogonal trajectories. The components of the metric tensor for this system are given by $g^{11}=\left(\partial f^{1} / \partial x^{1}\right)^{2}+\left(\partial f^{1} / \partial x^{2}\right)^{2}, g^{12}=g^{21}=0, g^{22}=\left(\partial f^{2} / \partial x^{1}\right)^{2}+\left(\partial f^{2} / \partial x^{2}\right)^{2}, g^{11}=$ $1 / g_{11}, g^{22}=1 / g_{22}$, and $g_{12}=g_{21}=0$. In terms of these quantities, the covariant components of a unit normal to the curves $f^{1}\left(x^{1}, x^{2}\right)=$ constant are given by

$$
n_{1}=\left(g^{11}\right)^{-1 / 2} \text { and } n_{2}=0 .
$$

The contravariant components are given in terms of the covariant components by the relation $n^{i}=g^{i i} n_{i}$, so that

$$
n^{1}=\left(g^{11}\right)^{1 / 2} \text { and } n^{2}=0 .
$$

In this coordinate system, Eqs. (6a) and (6b) are given by

$$
\begin{aligned}
& (p-\rho \Phi)_{, 1}=\frac{1}{4} G_{2}(0) \partial / \partial f^{1}\left(\log g_{22}\right) \\
& (p-\rho \Phi)_{, 2}=\frac{1}{4} G_{2}(0) \partial / \partial f^{2}\left(\log g_{11}\right) .
\end{aligned}
$$

Integrating these equations and equating the results, we obtain

$$
\log g_{11} g_{22}=H_{1}\left(f^{1}\right)+H_{2}\left(f^{2}\right)
$$

so that the product $g_{11} g_{22}$ is given by

$$
g_{11} g_{22}=e^{H_{1}\left(f^{1}\right)} \cdot e^{H_{2}\left(f^{2}\right)} \text {. }
$$

Now, let us make the coordinate transformation $u^{1}=u^{1}\left(f^{1}\right), u^{2}=u^{2}\left(f^{2}\right)$ which is defined by the relations $\left[d f^{1} / d u^{1}\right]^{2}=e^{-H_{1}\left(f^{2}\right)}$ and $\left[d f^{2} / d u^{2}\right]^{2}=e^{-H_{2}\left(f^{2}\right)}$ so that the components of the metric of the new system are given by

$$
a_{11}=g_{11} e^{-H_{1}\left(\delta^{1}\right)}, \quad a_{22}=g_{22} e^{-H_{2}\left(s^{2}\right)}, \quad \text { and } \quad a_{12}=a_{21}=0 .
$$

In any coordinate system, Eq. (6c) is given by

$$
n_{, i}^{i}=A(2)^{1 / 2} / G_{1}(0)
$$

and in this system, it becomes

$$
\frac{1}{2}\left(a^{11}\right)^{1 / 2} a^{22} \partial a_{22} / \partial u^{1}=A(2)^{1 / 2} / G_{1}(0) .
$$

Letting $B=A(2)^{1 / 2} / G_{1}(0)$ and substituting Eqs. (13) and (14) in Eq. (16), we obtain, since $a^{11}=1 / \dot{a}_{11}$ and $a^{22}=1 / a_{22}$,

$$
\left(a_{22}\right)^{-1 / 2} \partial a_{22} / \partial u^{1}=2 B
$$

Integrating, we obtain

$$
a_{22}=\left[B u^{1}+P\left(u^{2}\right)\right]^{2} .
$$

Now, $u^{1}$ and $u^{2}$ are coordinates in the plane, so that we can make use of the requirement that the Riemann tensor vanish. Since, from Eqs. (13) and (14), $a_{11} a_{22}=1$, this requirement is satisfied, as is easily shown, if and only if the following partial differential equation is satisfied

$$
\partial^{2} a_{11} / \partial u^{2^{2}}+\partial^{2} a_{22} / \partial u^{12}=0 .
$$

From Eqs. (18) and (19), we obtain

$$
\partial^{2} a_{11} / \partial u^{22}=-2 B^{2} .
$$


Integrating twice, we obtain

$$
a_{11}=-B^{2} u^{2 *}+Q_{1}\left(u^{1}\right) u^{2}+Q_{2}\left(u^{1}\right) .
$$

Since $a_{11} a_{22}=1$ for all values of $u^{1}$ and $u^{2}$, we may write

$$
\partial^{3} a_{11} / \partial u^{1}\left(\partial u^{2}\right)^{2} \equiv \partial^{3} a^{22} / \partial u^{1}\left(\partial u^{2}\right)^{2} .
$$

Making use of Eqs. (18) and (19), this relation becomes

$$
6 B\left[B u^{1}+P\left(u^{2}\right)\right]^{-4} d^{2} P\left(u^{2}\right) / d u^{22}-24 B\left[B u^{1}+P\left(u^{2}\right)\right]^{-5}\left[d P\left(u^{2}\right) / d u^{2}\right]^{2}=0 .
$$

Since $a_{11}$ and $a_{22}$ are never zero, we multiply by $\left[B u^{1}+P\left(u^{2}\right)\right]^{5}$, so that Eq. (23) reduces to

$$
6 B\left[B u^{1}+P\left(u^{2}\right)\right] d^{2} P\left(u^{2}\right) / d u^{2}-24 B\left[d P\left(u^{2}\right) / d u^{2}\right]^{2}=0 .
$$

This relation is clearly satisfied for all $u^{1}$ and $u^{2}$, if and only if $B=0$ or $P\left(u^{2}\right)$ is a constant. However, when $P\left(u^{2}\right)=$ constant, it follows easily from Eqs. (18) and (21) and the condition $a_{11} a_{22}=1$ that $B=0$. Thus, Eq. (24) is satisfied if and only if $B=0$.

When $B=0$, it follows from Eq. (17) that $a_{22}$ is a function of $u^{2}$ only; hence since $a_{11} a_{22}=1, a_{11}$ must also be a function of $u^{2}$ only. We accordingly make the coordinate transformation defined by the relations $d u^{1} / d v^{1}=1$ and $d u^{2} / d v^{2}=\left(a_{22}\right)^{-1 / 2}$. The components of the metric tensor in this case are given by

$$
b_{11}=\left[b^{11}\right]^{-1}=T\left(u^{2}\right), \quad b_{12}=b^{12}=0, \quad \text { and } \quad b_{22}=\left[b^{22}\right]^{-1}=1 .
$$

In the $v^{1}, v^{2}$ coordinate system the condition that the Riemann tensor vanish is satisfied if and only if the following differential equation is satisfied.

$$
2 d^{2} b_{11} / d v^{2}=1 / b_{11}\left[d b_{11} / d v^{2}\right]^{2} .
$$

The two solutions to this differential equation are $b_{11}=$ constant and $b_{11}=\left[C v^{2}+D\right]^{2}$. Making a coordinate transformation of the form $w^{1}=\alpha_{1} v^{1}+\beta_{1}, w^{2}=\alpha_{2} v^{2}+\beta_{2}$, we can find $\alpha_{i}$ and $\beta_{i}$ such that the components of the corresponding metric tensor are $\bar{b}_{11}=1, \bar{b}_{22}=1$, and $\bar{b}_{11}=\left(w^{2}\right)^{2}, \bar{b}_{22}=1$ respectively for $b_{11}=$ constant and $b_{11}=$ $\left[C v^{2}+D\right]^{2}$. When $\bar{b}_{11}=1$, the $w^{1}, w^{2}$ coordinate system is Cartesian and when $\bar{b}_{11}=$ $\left[w^{2}\right]^{2}$, it is polar where the curves $w^{1}=$ constant are straight lines in both cases. Since each coordinate transformation made in this section has been of the form $t^{1}=t^{1}\left(s^{1}\right)$, $t^{2}=t^{2}\left(s^{2}\right)$, and the curves $w^{1}=$ constant are either parallel or concurrent straight lines, it follows that the curves of constant speed are either parallel or concurrent straight lines.

5. Existence conditions. Ericksen has shown for the case of steady rectilinear motion that the speed is not overdetermined when the two scalars which occur in Rivlin's equations are proportional. For this case we show that there are additional restrictions which must be imposed on these scalars in order that steady rectilinear motion be possible in every closed cylinder, when both the speed and stress are well behaved throughout the region of motion. Specifically, we require in every case that the rate of work $t_{i}^{i} d_{i}^{i}$ be non-negative; that the stress exist and be finite in the region of motion; that the speed of particles on the cylinder wall be zero; and that the speed be continuously differentiable in the region of motion.

For the case of steady rectilinear motion, parallel to the $x^{3}$ axis of a rectangular coordinate system where the speed is not overdetermined, Rivlin's equation for the 
stress is

$$
\begin{aligned}
\left\|t_{i i}\right\|=-p\left\|\delta_{i j}\right\|+F_{1}(\mathrm{II})\left\|\begin{array}{ccc}
0 & 0 & f_{.1} \\
0 & 0 & f_{.2} \\
f_{.1} & f_{.2} & 0
\end{array}\right\| \\
+c F_{1}(\mathrm{II})\left\|\begin{array}{ccc}
f_{.1}^{2} & f_{.1} f_{.2} & 0 \\
f_{.1} f_{.2} & f_{.2}^{2} & 0 \\
0 & 0 & f_{.1}^{2}+f_{.2}^{2}
\end{array}\right\| .
\end{aligned}
$$

As an immediate consequence of the assumed behavior of the stress and speed, it follows from Eq. (27) that $F_{1}$ (II) is finite at all points where $-\mathrm{II}=f_{.2}^{2}+f_{.1}^{2}$ does not vanish

We now consider the behavior of $F_{1}$ (II) at those points in the region of motion where both partial derivatives of $f$ vanish; namely, when II vanishes. That such points exist is guaranteed by the assumptions that $f$ is continuously differentiable throughout the region of motion and is zero on the cylinder wall and by the theorem which states that any continuous function which is constant on the boundary of its region of definition is either constant or has both a maximum and a minimum in this region. If we exclude the case where $f$ is constant and make use of the requirement that the stress exist and be finite throughout the region of motion, then it follows from Eq. (27) that $\lim _{I I \rightarrow 0} F_{1}(\mathrm{II}) f_{.1}$ and $\lim _{\mathrm{II} \rightarrow 0} F_{1}(\mathrm{II}) f_{.2}$ exist and are finite. When this is the case, it follows that

$$
\left\{\left[\lim _{\mathrm{II} \rightarrow 0} F_{1}(\mathrm{II}) f_{.1}\right]^{2}+\left[\lim _{\mathrm{II} \rightarrow 0} F_{1}(\mathrm{II}) f_{.2}\right]^{2}\right\}^{1 / 2}=C \quad \text { (a constant). }
$$

Eq. (28) can be written in the equivalent form

$$
\lim _{\mathrm{II} \rightarrow 0}(-\mathrm{II})^{1 / 2} F_{1}(\mathrm{II})=C .
$$

When $C$ is zero, then $\lim _{\mathrm{II} \rightarrow 0} F_{1}$ (II) $f_{, 1}=0$ for $i=1,2$ when $C \neq 0$, then $\lim _{\mathrm{II} \rightarrow 0}$ $F_{1}(\mathrm{II}) f_{, i}=$ constant for $i=1,2$. Multiplying the expression $F_{1}(\mathrm{II}) f_{, i}$ by $\left(-\mathrm{II}^{1 / 2}\right)$ $(-\mathrm{II})^{-1 / 2}$, we obtain.

$$
F_{1}(\mathrm{II})(-\mathrm{II})^{1 / 2} \cdot f_{. i}(-\mathrm{II})^{-1 / 2} .
$$

Making use of Eq. (29) and taking the limit as II goes to zero, we obtain

$$
\lim _{I I \rightarrow 0} f_{. i}(- \text { II })^{-1 / 2}=\text { constant. }
$$

Eq. (31) represents the requirement that the components of the unit normal to the curves of constant speed must exist at points where II vanishes. An indication that this condition can not be satisfied for $C \neq 0$ is given in the case of radially symmetric Poiseuille flow [5]. In polar coordinates, the differential equation determining the speed, $f$, is

$$
d f / d r F_{1}\left([d f / d r]^{2}\right)=-D r
$$

where $D$ is a constant. By symmetry, and since $f$ is continuously differentiable, $d f / d r$ clearly vanishes at $r=0$. In this case, $-I I=(d f / d r)^{2}$ so that Eq. (32) cannot be satisfied unless $\lim _{\mathrm{II} \rightarrow 0} F_{1}$ (II) $(-\mathrm{II})^{1 / 2}=0$. When this limit is zero, Eq. (32) can be satisfied. For example, when $F\left([d f / d r]^{2}\right)$ is given by $K(d f / d v)^{-1 / 2}$ a solution to Eq. 
(32) is

$$
f=A r^{3}+B
$$

where $A$ and $B$ are constants.

The reader should note here that this result does not justify requiring that the constant in Eq. (29) be zero, since it is assumed that the flow is radially symmetric, and we do not show that this is the only type of Poiseuille flow.

In section 2 of this paper, we showed that few types of steady rectilinear motion were possible when $F_{1}$ (II) is of the form $K(-\mathrm{II})^{-1 / 2}$, in which case the constant, $C$, defined in Eq. (29) is non-zero. Accordingly, if we expect steady rectilinear flow to be possible in every closed smooth cylinder, when the stress and speed behave as assumed in this section, then the scalar $F_{1}$ (II) must be expressible in the form

$$
F_{1}(\mathrm{II})=K(-\mathrm{II})^{-1 / 2}+Y(\mathrm{II})
$$

where $K$ is a non-zero constant and $Y$ is a function for which $\lim _{\mathrm{II} \rightarrow 0}(-\mathrm{II})^{1 / 2} Y(\mathrm{II})=0$, and is finite at all points where II does not vanish.

6. Conclusion. If there are classes of Rivlin fluids for which steady rectilinear motion is possible in every closed smooth cylinder when the stress and speed satisfy the assumptions listed in the previous section, then these materials must satisfy certain definite conditions. These conditions are: (1) the stress be affected by uniform changes in the speed; and (2) the scalar $F_{1}$ (II) must be expressible in the form $A(-\mathrm{II})^{-1 / 2}+$ $Y(\mathrm{II})$ where $A$ is a non-negative constant and $Y(\mathrm{II})$ is a function which is finite when II $\neq 0$ and for which $\lim _{\mathrm{II} \rightarrow 0}(-\mathrm{II})^{1 / 2} Y(\mathrm{II})=0$.

7. Acknowledgment. The author wishes to express his gratitude to Dr. J. L. Ericksen of the Naval Research Laboratory for suggesting this paper and for his guidance in carrying out the work.

\section{REFERENCES}

[1] R. S. Rivlin, Hydrodynamics of Non-Newtonian Fluids, Nature 160, 611-613 (1947).

[2] J. L. Ericksen, Overdetermination of the Speed in Rectilinear Motion of Non-Nertonian Fluids, Quarterly of Applied Mathematics (forthcoming).

[3] A. E. Green and R. S. Rivlin, Steady Flow of Non-Newtonian Fluids through Tubes, Department of the Army (Ordnance Corps) DA-3487/2, Brown University report, Oct. 1955.

[4] R. Courant, Differential and Integral Calculus, Interscience, 1937.

[5] C. Truesdell, The Mechanical Foundations of Elasticity and Fluid Dynamics, J. Rational Mechanics and Analysis 1 (1952). 